\title{
Time-Domain Sensing of Targets Buried Under a Rough Air-Ground Interface
}

\author{
Traian Dogaru, Student Member, IEEE, and Lawrence Carin, Senior Member, IEEE
}

\begin{abstract}
We consider plane wave time-domain scattering from a fixed target in the presence of a rough (random) surface with application to ground penetrating radar. The timedomain scattering data are computed via a two-dimensional (2-D) finite-difference time-domain (FDTD) algorithm. In addition to examining the statistics of the time-domain fields scattered from such a surface, we investigate subsurface target detection by employing a (commonly used) matched-filter detector. The results of such a detector are characterized by their receiver operating characteristic (ROC), which quantifies the probability of detection and probability of false alarm. Such ROC studies allow us to investigate fundamental assumptions in the matched-filter detector: that the target response is deterministic and the clutter signal stochastic, with the two signals treated as additive and independent.
\end{abstract}

Index Terms-Buried object detection, time-domain analysis.

\section{INTRODUCTION}

$\mathbf{O}$ VER the last few decades, significant research has been undertaken on the scattering of electromagnetic and acoustic waves from rough (random) surfaces [1]-[9] with the scattered-field properties parametrized statistically. Initial work in this area was based on approximate formulations, while more recently, there has been a significant focus on the application of numerical algorithms. In most of these analytical and numerical analyses, frequency-domain operation has been considered with results presented as the mean and variance of the angular-dependent scattered field. There has been very little work done on time-domain operation (although some results were calculated using time-domain algorithms [2]) or on the characterization of the field scattered from a deterministic target in the presence of a rough (random) interface.

There are many applications for which one would be interested in detecting/identifying a target in the presence of a rough surface. In electromagnetics, one may encounter the problem of detecting/identifying a low-flying aircraft over ocean or a buried target under a rough air-ground interface. Similar problems can be posed in acoustics. In this paper, we consider the case of a deterministic (fixed) target in the presence of a rough surface, the latter being parametrized statistically. This study is motivated by electromagnetic sensing of buried targets

Manuscript received February 7, 1997; revised October 21, 1997. This work was supported in part by the Army Research Office under Grant DAAH04-961-0448, Demining MURI, by the Army Research Laboratory, Adelphi, MD, by the Air Force Office of Scientific Research under Grant F49620-94-1-0363, and by the Office of Naval Research under Grant N00014-96-1-0861.

The authors are with the Department of Electrical and Computer Engineering, Duke University, Durham, NC 27708 USA.

Publisher Item Identifier S 0018-926X(98)02258-3.
[10]-[17] (e.g., mines, unexploded ordnance, etc.), while, as alluded to above, this general problem has wide applicability.

For electromagnetic scattering from the ocean surface, a stochastic surface parametrization is clearly required since the sea-surface characteristics generally change with time in a seemingly random fashion. However, for the sensing of an underground target, the air-ground interface is fixed (deterministic), calling into question the need for a statistical analysis. However, although a given portion of the air-ground interface is fixed, it will, in general, be different from that of another (fixed) region of the interface. Thus, while the fields scattered from any particular portion of the rough surface are deterministic, to characterize the fields scattered from such a general surface, the surface (roughness) parameters must be characterized statistically and, therefore, so must the associated scattered fields. Consequently, to characterize the fields scattered from such a rough air-ground interface, the surface properties are modeled as a random process with any particular surface constituting one realization of an ensemble, each of which is parametrized by the same probability density function. In turn, the scattered fields from such a surface are also treated as a random process.

As discussed above, most previous analyses of scattering from rough surfaces have been performed in the frequency domain. However, there has been significant interest recently in time-domain ground-penetrating radar (GPR) systems [10]-[19] motivating the work presented here on timedomain scattering. The time-domain field scattered from a rough (random) surface constitutes a random process, which, in general, is nonwhite. Therefore, for detecting a target in the vicinity of such a rough surface, optimal detectors [20] (under appropriate conditions) are preceded by a whitening filter [20] such that the clutter becomes a white (uncorrelated) time sequence. The whitening filter employs the clutter's correlation matrix, which for wide-sense stationary (WSS) clutter can be represented in the Fourier domain in terms of the clutter's power spectral density (PSD). As discussed below, in the cases we have examined thus far, the WSS model has been found to be quite accurate and, therefore, here we quantify the transient clutter statistics via the PSD.

Although the statistical properties of time-domain scattering from a rough (random) surface are of interest in their own right and are investigated here in the context of their PSD, the principal focus of the present paper is a detailed examination of the underlying assumptions used in classical optimum detectors with emphasis on time-domain GPR. Matched filters preceded by a whitening filter are commonly utilized for the 
detection of a known target in noise/clutter. For the case of GPR, such detectors are useful because there are generally a relatively small number of target types (e.g., mines) of interest, the signatures of which can in principle be measured or computed. In its simplest (and most common) manifestation, such a matched filter assumes that the target signature is deterministic and known exactly with the clutter/noise treated as stochastic and additive. While such properties result in a simple detector, in practice they are often not rigorously valid. To test such a detector and its underlying assumptions, our results are presented in the form of receiver operating characteristic (ROC) curves, which describe how the probability of false alarm varies with the probability of detection (as detailed below, these probabilities are varied by adjusting the detector threshold). Using classical detection theory [20], theoretical ROC curves are computed based on the clutter statistics. Monte Carlo simulations are then performed for a target in the presence of a random surface to get "empirical" detector performance. Where the theoretical and empirical ROC curves agree, we deem the underlying detector assumptions valid. Of interest is the discrepancy in the theoretical and empirical ROC curves as a function of target position, angle of incidence, and surface statistics. Such studies are important for when the simplifying assumptions are inappropriate, more sophisticated detectors, which, for example, exploit the random character of the target signature [20], may yield better performance than the simple matched filter (at the price of greater complexity).

To perform the aforementioned study, many realizations of the air-ground interface must be considered (each described by the same probability density function) and, therefore, the numerical scattering model must be versatile and highly efficient. Consequently, all results are computed using a twodimensional (2-D) finite-difference time-domain (FDTD) algorithm [21]-[23], which incorporates a lossy half space, surface roughness, plane-wave incidence, a near-to-far-zone transformation, and a perfectly matched layer (PML) absorbing boundary condition (ABC) [24]-[29]. Acquiring statistics from a three-dimensional (3-D) surface would require prohibitive computational resources; however, it is felt that the basic issues studied here using a 2-D model are also relevant to the more realistic case of 3-D GPR systems.

The remainder of the text is organized as follows. A brief discussion of the FDTD algorithm is given in Section II with careful attention directed on the near-to-far-zone transformation. The matched-filter detector and whitening filter are discussed in Section III, wherein basic underlying assumptions are summarized. Results are presented in Section IV for several realizations of a target in the presence of a rough (random) air-ground interface, presented in the form of PSD's for the clutter and ROC curves for the matched-filter detector. This work is summarized and conclusions are given in Section V.

\section{NUmerical SCATtering MOdel}

\section{A. Basic Numerical Algorithm}

All numerical scattered-field data are calculated via a 2-D FDTD code, utilizing the classic Yee algorithm [21]. Results
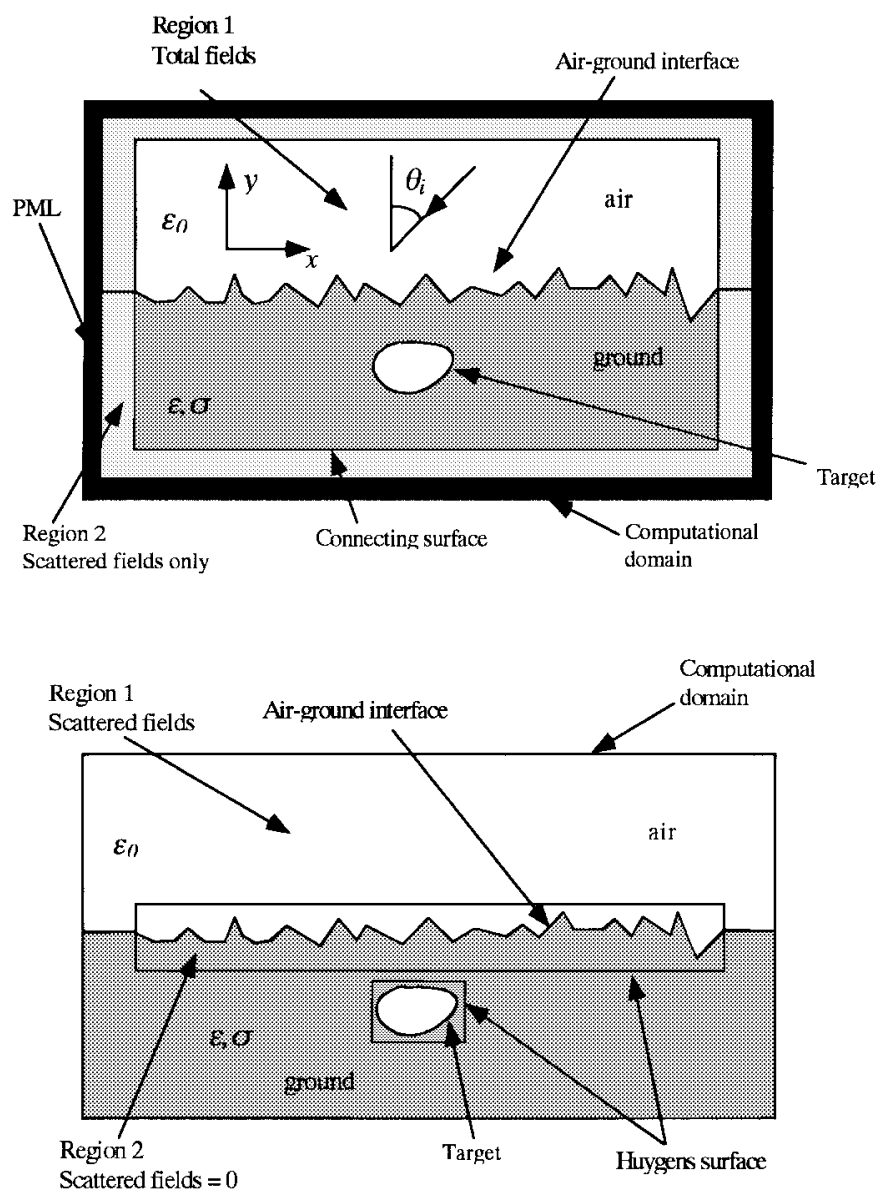

Fig. 1. Schematic of (a) FDTD computational model and (b) the Huygens surfaces used to calculate the far-zone scattered fields.

are presented for $\mathrm{TM}_{z}$ polarization $\left(H_{z}=0\right)$ with similar results found (but not presented) for the $\mathrm{TE}_{z}\left(E_{z}=0\right)$ case (see Fig. 1). As described in [25] and [26], the plane wave incident field is modeled via the use of Huygens currents placed along a closed contour with total fields inside and scattered fields outside. We refer to "the incident field" as the plane wave that impinges on the half-space plus the fields reflected and transmitted at the planar interface in the absence of any target or surface roughness. The scattered field is produced by any perturbations to this half-space problem (target and/or surface roughness).

At the boundaries of the computational domain, we use a PML [27] to absorb the outwardly propagating scattered fields. It has been well established that the Berenger [27] PML yields a reflection coefficient at the boundary of the computational domain that is orders of magnitude smaller than absorbing boundary conditions derived from the one-way wave equation [30]. However, the PML introduced by Berenger in his original paper cannot be applied directly to lossy media and, therefore, we use a PML formulation based on [28] and [29] appropriate for lossy soils.

The far-zone scattered fields are the quantities of interest in most radar problems. Therefore, we implement a near-to-farzone transformation in a manner similar to [26]. In particular, the perturbations (target and/or rough surface) are enclosed by Huygens surfaces and the equivalent currents thereon 
(minus the incident field) are integrated with an analytic approximation [31] to the half-space Green s function. As shown in Fig. 1, separate near-to-far-zone Huygens surfaces are used for the rough surface and the target (when present). The fields are observed in backscatter for the near-grazing case applicable to many airborne subsurface radars [18].

Near-to-far-zone transformations have been used for many years to propagate FDTD computed near-zone fields into the far zone. However, much of this work has been for free-space scattering with far fewer results presented for the half-space problem [26] considered here. Therefore, we elaborate on several points germane to the half-space problem recognized in the course of this research. First, as discussed above, the far-zone fields are computed via an approximate half-space Green's function derived via an asymptotic evaluation of its exact Sommerfeld-integral representation [31]. In all such asymptotic analyses, one must first identify the highly oscillatory and relatively slowly varying portions of the integral. The Sommerfeld integrals are evaluated approximately via saddlepoint integration in the vicinity of its stationary point(s) [31], the latter determined by the phase of the highly oscillatory terms [31]. For evaluation of the scattered fields above the halfspace far from the interface, one usually treats the exponentials characteristic of propagation in air as the highly oscillatory terms and the remaining exponentials that describe propagation in the dielectric half-space as slowly varying. However, if the currents in the dielectric are at electrically large depths, a more accurate asymptotic representation of the Green s function may be necessary. In particular, some of the aforementioned terms may no longer be deemed slowly varying in which case they must be included when determining the stationary point(s) [32]. This issue is particularly relevant for GPR applications in which the dielectric constant of the soil is often large and, therefore, the target need not be too deep physically for the above issues to be of concern (this is especially true for the wide-band signals of interest here).

A second issue involves numerical dispersion [33], [34]. As the time-domain fields propagate through the FDTD grid, there is a inherent (nonphysical) pulse distortion caused by the difference-equation approximations to Maxwell s equations [33], [34]. This issue is exacerbated for electrically large structures, of interest for the high-dielectric-constant halfspace region. Numerical dispersion is mitigated by increasing the spatial sampling in the FDTD grid, but the physical size of the problem and finite computer memory ultimately dictate the discretization limits. Therefore, to improve the accuracy of the far-zone fields, we have found it useful to place the Huygens surface as close as possible to the target to minimize the range over which FDTD computed scattered fields must propagate (of course, similar concerns are relevant for the incident-field Huygens surface as well).

For the target depths and soil properties considered in Section IV, the simple Green's function approximation has proven very accurate after performing exhaustive tests. However, similar tests also showed numerical dispersion to be a concern, requiring one to be very careful about the spatial discretization and requiring the Huygens surface to circumscribe the target (and rough surface) tightly. In the results presented below, the spatial discretization was 12 samples per wavelength (in the soil), at the highest frequency of interest in the incident fields. Petropoulos [33], [34] has demonstrated that the required sampling rate is predicated by both the dielectric constant and the length over which the FDTD fields must propagate. We are confident in the FDTD and near-to-farzone results presented below, but it must be emphasized that this confidence has been obtained after a rigorous examination of numerical dispersion and its pitfalls.

\section{B. Rough-Surface Generation}

For the GPR problem, clutter can be generated from multiple sources, including surface roughness and/or subsurface inhomogeneities (e.g., rocks, roots, inhomogeneous soil, etc.). In this paper, we restrict ourselves to clutter generated by surface roughness under the assumption that the subsurface is characterized by a homogeneous lossy dielectric. Although other or additional forms of clutter may occur in practice, there has been very little work done to date on wide-band time-domain scattering from rough surfaces or from targets situated in such environments. Moreover, as discussed in Section V, the general insight from this problem may yield an appreciation for the physics inherent in more general clutter scenarios.

We assume that the surface height $f(x)$ at each transverse location $x$ is a zero-mean Gaussian random variable with heights $f(x)$ and $f(x+\Delta x)$ related by the autocorrelation

$$
E\left[(f(x) f(x+\Delta x)]=h^{2} \exp \left(-\Delta x^{2} / l^{2}\right)\right.
$$

where $h^{2}$ is the variance, $l$ is the correlation length, and $E(\cdot)$ represents the expectation operator. In actual implementation, the random process is generated in the Fourier domain [7] by passing a Gaussian white-noise process through a filter with a spatial-frequency response corresponding to the desired rough-surface power spectrum [the Fourier transform of (1)].

As described in Secion II-A, we calculate the scattered fields using a standard, 2-D Yee FDTD algorithm [21]. In such a scheme, the rough surface must be approximated in a staircase fashion [2], [21] due to the Cartesian spatial discretization. However, it has been demonstrated that if the spatial gridding is sufficiently fine, the scattered fields are in very good agreement with those calculated via a more sophisticated FDTD algorithm in which the surface roughness is modeled using a contour-integral procedure [2].

\section{Time-Domain Statistics And Detection}

\section{A. Time-Domain Statistics}

Frequency-domain scattering from rough (random) surfaces has been characterized in terms of the mean and variance of the scattered fields [1]-[9]. This parametrization is natural since at a given frequency the scattered field is a random variable. However, in the time domain, the scattered field is a time-dependent random process. For all the cases we have considered in the course of this work using the surface roughness in (1), the time-domain fields scattered from the rough surface are approximately wide-sense stationary (the 
correlation matrix [35] computed via averaging hundreds of Monte Carlo realizations has Toeplitz symmetry with less than $5 \%$ error). Therefore, we describe the statistical properties of the rough-surface scattered field in terms of its autocorrelation [35]. One drawback of this procedure is that the autocorrelation is dependent on the incident-pulse temporal shape $g(t)$, viz., if $r_{\delta}(t)$ represents the autocorrelation of the scattered fields when the incident pulse shape is a delta function, then the autocorrelation of the scattered field for the input-pulse $g(t)$ is

$$
r_{g}(t)=\int_{-\infty}^{\infty} \int_{-\infty}^{\infty} d \gamma d \beta g(\gamma) g(\beta) r_{\delta}(t-\beta+\gamma)
$$

The power-spectral density (PSD) of the scattered field $S_{g}(\omega)$ is represented by the Fourier transform of $r_{g}(t)$ and is expressed as

$$
S_{g}(\omega)=|G(\omega)|^{2} S_{\delta}(\omega)
$$

where $S_{\delta}(\omega)$ and $G(\omega)$ are the Fourier transforms of $r_{\delta}(t)$ and $g(t)$, respectively. Therefore, although the autocorrelation and PSD of the field scattered from a rough (random) surface are dependent on the incident-pulse shape, with knowledge of $G(\omega)$, one can calculate the incident-pulse-independent PSD $S_{\delta}(\omega)$ characteristic of the clutter alone.

\section{B. Optimal Detector}

In addition to studying the transient fields scattered from a rough (random) surface, a major focus of this investigation involves the detection of a fixed target in the vicinity of such a surface. As discussed in Section I, this is a basic problem of interest for many applications. Detection theory has been investigated for decades [20] and, therefore, the purpose here is not to develop a new detector. However, in classical detection theory, one often makes assumptions that simplify detector design. In practice, such detectors often do not work as well as expected and it is of significant interest to understand why, such that improved detectors can be developed, if necessary. The FDTD allows us to perform controlled experiments with which underlying detector assumptions can be investigated systematically here with application to GPR.

A concise summary of simple detection theory [20] is given, such that the underlying assumptions are understood for subsequent examination. We consider a binary test for hypotheses $H_{0}$ and $H_{1}$, where $H_{0}$ states that the scattered signal consists of clutter alone and $H_{1}$ that the signal consists of the superposition of clutter and the deterministic response from a known target. Implied in this test are the assumptions that: 1) there are only two possibilities for the source of the scattered field (i.e., that the binary hypothesis test is valid); 2) the target and clutter signatures are additive; and 3) the target has a deterministic signature. Assumption 1) constitutes a simplification of the general problem of multiple hypothesis testing [20] and allows a direct examination of 2) and 3). Our numerical experiment is easily designed such that 1 ) is valid. Therefore, we undertake a detailed examination of 2) and 3), which are of importance for multiple hypothesis testing as well.
Under the above hypotheses, a given polarization of scattered field $e(t)$ can be expressed as $e(t)=c(t)$ under hypothesis $H_{0}$ and $e(t)=E^{1 / 2} s(t)+c(t)$ under $H_{1}$, where $E^{1 / 2} s(t)$ is the known deterministic target signature (with energy $E$ ) and $c(t)$ is the stochastic clutter characterized by a joint probability density (in the remainder of the text when $E$ appears alone, it represents the energy of the deterministic scattered field and when it appears as $E(\cdot)$ it represents expectation). If the clutter $c(t)$ is white $\left(E\left[c\left(t_{i}\right) c\left(t_{k}\right)\right]=\right.$ $\delta_{W}^{2} \delta\left(t_{i}-t_{k}\right)$, where $\sigma_{W}^{2}$ is the variance if $c(t)$ is a zeromean process, then the optimal detector is a matched filter [20], [36]. In general, the clutter is not white and the optimal detector consists of a matched filter preceded by a whitening filter [20], [37]. If $h_{w}(t)$ represents the impulse response of the linear time-invariant whitening filter, then the new noise response $c_{*}(t)$ and signal response $s_{*}(t)$ after whitening are

$$
\begin{aligned}
& c_{*}(t)=\int_{-\infty}^{\infty} c(\tau) h_{w}(t-\tau) d \tau \\
& s_{*}(t)=\int_{-\infty}^{\infty} s(\tau) h_{w}(t-\tau) d \tau .
\end{aligned}
$$

If hypothesis $H_{0}$ is true, the filter output is $e_{*}(t)=c_{*}(t)$, while if $H_{1}$ is true, $e_{*}(t)=E^{1 / 2} s^{*}(t)+c_{*}(t)$. Finally, the output of the whitening filter is projected onto the function $E^{1 / 2} s_{*}(t)$ resulting in the random variable $l$

$$
l=\int_{-\infty}^{\infty} \sqrt{E} s_{*}(t) e_{*}(t) d t
$$

If $p\left(l \mid H_{0}\right)$ is the probability of $l$ under hypothesis $H_{0}$ and $p\left(l \mid H_{1}\right)$ is the probability of $l$ under $H_{1}$, the decision as to whether a target is present is effected via the statistic

$$
\Lambda=\frac{p\left(l \mid H_{1}\right)}{p\left(l \mid H_{0}\right)}=\frac{\exp \left[-\frac{(l-m)^{2}}{2 \sigma^{2}}\right]}{\exp \left[\frac{-l^{2}}{2 \sigma^{2}}\right]}
$$

where the right side of (6) is valid if $c_{*}(t)$ is a zero-mean Gaussian process (it is approximately white as a consequence of the whitening filter) with $m=E^{1 / 2}$ and $\sigma^{2}$ representing the variance of $c_{*}(t)$. For a given threshold $T$, we choose $H_{1}$ if $\Lambda>T$ and $H_{0}$ if $\Lambda<T$ [20]. If $c_{*}(t)$ is a zero-mean white Gaussian process, it is easy to see from the right side of (6) that the statistical properties of $\Lambda$ are dependent entirely on the statistics of $l$ and, therefore, the latter is termed a sufficient statistic [20] (and $l$ alone can be compared against a threshold).

For the surface characteristics in (1) and the examples studied here, we have found $c(t)$ to be well characterized by a Gaussian density function (this is not surprising, as dictated by the central limit theorem [35]). Therefore, we specialize the discussion to the Gaussian case. The detector performance is parametrized by computing the probability of a false alarm at threshold $T$

$$
P_{F}(T)=\operatorname{Pr}\left(l>T \mid H_{0}\right)=\int_{T}^{\infty} p\left(l \mid H_{0}\right) d l
$$

as well as the probability of detection

$$
P_{D}(T)=\operatorname{Pr}\left(l>T \mid H_{1}\right)=\int_{T}^{\infty} p\left(l \mid H_{1}\right) d l .
$$


Detectors are usually characterized by plotting the $P_{F}$ and $P_{D}$ as a function of the threshold $T$ and this representation is termed the receiver operating characteristic (ROC) [20]. One can adjust the parameter $T$ to operate the detector at a $P_{F}$ and $P_{D}$ that is appropriate for a given application.

Summarizing, we will use the classical (whitening-filter)(matched-filter) detector discussed above to investigate the radar detection of subsurface targets. Other more-complicated detectors could be used if the aforementioned assumptions breakdown, although this results in a significant escalation in detector complexity [20]. With regard to the results presented below, the whitening filter is implemented via a forward (linear-prediction) error filter [37], the parameters of which are computed by solving the classical Wiener-Hopf equations [37], which require the autocorrelation matrix of the clutter $c(t)$ (in the absence of the target). The autocorrelation matrix was computed by averaging the scattered fields from 300 realizations of the rough surface, each of which is described by the same rough-surface density function.

\section{RESULTS}

For all examples presented here, the excitation is in the form of an obliquely incident plane wave with incident pulse shape and spectrum shown in Fig. 2. This waveform is representative of signals generated routinely by ultra-wide-band SAR systems [17]-[19]. While we consider various statistical models for the rough-surface properties, the electrical characteristics of the soil itself are kept fixed. In particular, the soil is modeled with a frequency-independent dielectric constant $\epsilon_{r}=6$ and conductivity $\sigma=0.005 \mathrm{~S} / \mathrm{m}$. While this model does not correspond to any particular soil, it is consistent with data measured for various soil types of interest [38]. Note that although the dielectric constant and conductivity are frequency independent in this model, the incident and scattered waveforms are dispersed as they propagate through the soil due to the frequency dependence of the index of refraction $n(\omega)=\left[\epsilon_{r}-j \sigma / \omega \epsilon_{o}\right]^{1 / 2}$. Thus, the equivalent (Huygens) currents used to model the incident plane wave were computed in the frequency domain before Fourier conversion to the timedomain form used in the FDTD algorithm. The 2-D target considered has a square cross section $(37.5 \mathrm{~cm} \times 12.5 \mathrm{~cm})$ and is composed of a homogeneous lossless dielectric. Moreover, the top of the target is buried $25 \mathrm{~cm}$ beneath the mean position of the rough air-ground interface. The dielectric target was considered because it presents a particularly challenging case for radar systems.

In the following results, the rough-surface profile in (1) has been used, with various parameters for the standard deviation of the surface height $h$ and the correlation length $l$. Moreover, two angles of incidence are considered. Finally, with regard to the target, although its shape and depth are fixed, we consider several examples for its relative dielectric constant. The purpose of this study is to explore basic target (rough surface) interactions and their impact on the performance of a common detector (the matched filter). We note, however, that there are clearly other possibilities for the surface roughness statistics, target type, and soil type. Such issues will be considered in future work.

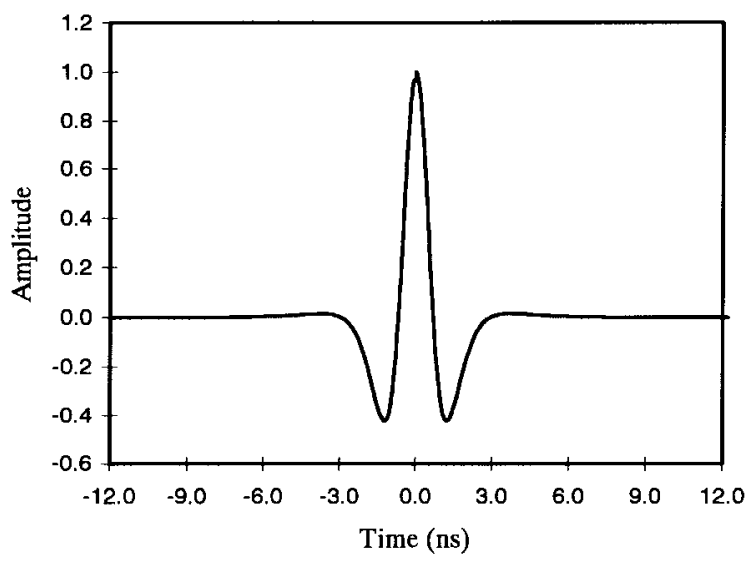

(a)

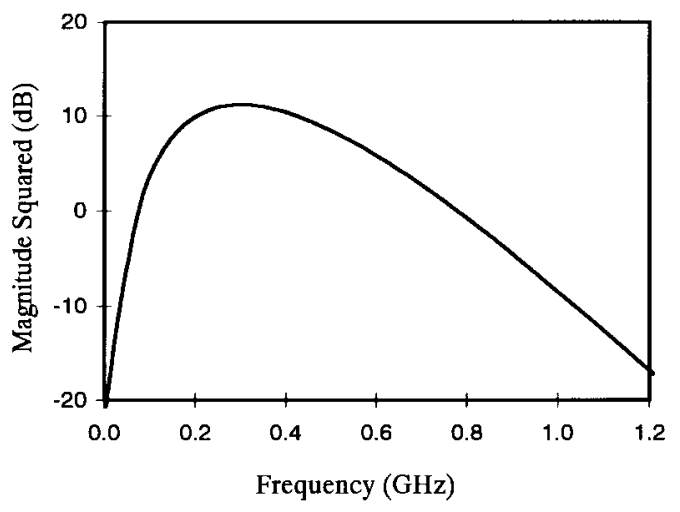

(b)

Fig. 2. (a) The incident pulse and (b) its spectrum used in all scattered-field computations.

Before proceeding, recall that for the examples considered here, we have assumed plane wave incidence. This is motivated by the SAR problem [17]-[19] for which the sensor is quite distant from the region under interrogation. Other authors have considered a tapered excitation [2] in the FDTD computations, which may be representative of the antenna pattern for some applications; moreover, such tapers mitigate numerical difficulties at the edges of the rough surface from which fields are diffracted in a manner uncharacteristic of the surface itself. While this latter issue is important for the computation of rough-surface statistics, it is not necessary for the detection examples considered here. In particular, note from (5) that the decision test statistic $l$ is computed by projecting the total measured waveform $e_{*}(t)$ onto $s_{*}(t)$ - the latter representing the target signature for a flat surface. Therefore, nonzero values of $s_{*}(t)$ only exist over a very limited temporal support (for the targets considered here) and, therefore, the only portion of $e_{*}(t)$ of interest for computation of $l$ is that for which $s_{*}(t)$ is nonzero. In our computations, we have carefully examined diffractions from the edges of the rough surface and these occur well outside the meaningful temporal support of $e_{*}(t)$. We note as well that the clutter statistics (used in the whitening filter) were also computed by considering those portions of $c_{*}(t)$ with support around 


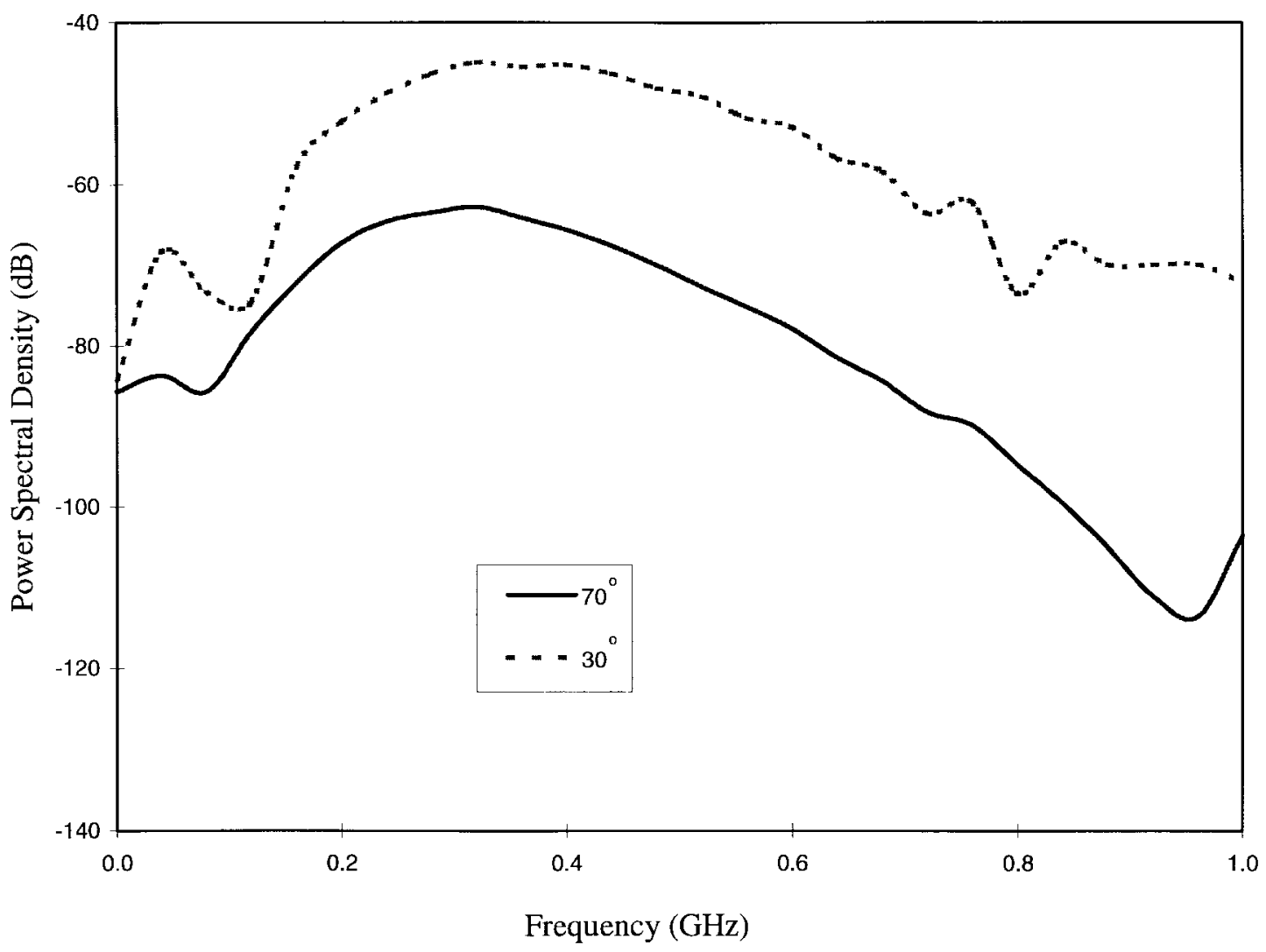

Fig. 3. Power spectral density (PSD) of the transient fields backscattered from the rough surface in (1), using the incident pulse in Fig. 2. The Gaussian rough surface has correlation length $l=18.75 \mathrm{~cm}$ and standard deviation $h=3.95 \mathrm{~cm}$ and the soil is characterized by $\epsilon_{r}=6$ and $\sigma=0.005 \mathrm{~S} / \mathrm{m}$. Results are plotted for angles of incidence $\theta_{i}=30^{\circ}$ and $70^{\circ}$.

the principal portion of $e_{*}(t)$. Therefore, edge-diffractioninduced vitiation of the detector computations was avoided by exploiting the temporal filtering of the short-pulse time-domain excitation.

\section{A. Clutter Characteristics}

As discussed in Section III-A, the clutter statistics are characterized by their power spectral density (PSD) - assuming the clutter is wide-sense-stationary, as we have found to be approximately the case for all examples considered here. In Fig. 3 we address the variation in the PSD with respect to the angles $\theta_{i}=30^{\circ}$ and $70^{\circ}$ (see Fig. 1), for a surface roughness characterized by $h=3.95 \mathrm{~cm}$ and $l=18.75 \mathrm{~cm}$. The most striking feature of these results is the significant decrease in the strength of the PSD as one gets closer to grazing, which is expected due to the fact that the backscattered fields excited by the rough surface diminish in strength as one approaches grazing. As a comparison with the PSD's, we consider the spectrums of waveforms backscattered from a buried target, for the same angles considered in Fig. 3. In Fig. 4, we plot the angular-dependent backscatter spectrums for a $37.5 \mathrm{~cm}$ $\times 12.5 \mathrm{~cm}$ target of $\epsilon_{r}=2$ buried $25 \mathrm{~cm}$ under a flat air-ground interface. For the target considered here, we see from Fig. 4 that the shape of the backscatter spectrum is relatively insensitive to angle, while, as in Fig. 3, there is a pronounced reduction in its strength as one approaches grazing. To avoid problems inherent in deconvolving the incident pulse, the spectrums in Figs. 3 and 4 represent deltafunction responses filtered by the incident pulse in Fig. 2 [see discussion concerning (2) and (3)].

The remaining results will be presented in the form of ROC curves, which depict the variation in the probability of detection $P_{D}$ and probability of false alarm $P_{F}$ as a function of the detector threshold $T$ (see Section III-B). For the case of additive white Gaussian noise, detector performance $\left(P_{D}\right.$ and $\left.P_{F}\right)$ is dictated entirely by the parameter $d=m / \sigma$ [20], where from Section III-B $m^{2}$ is the signal energy and $\sigma^{2}$ is the variance of the clutter. Consequently, a reduction in the strength of the backscattered signature does not necessarily imply that detector performance will diminish. In fact, if the clutter variance reduces as well, then it is possible that the ratio $d=m / \sigma$ may actually increase (note in Figs. 3 and 4 the commensurate decrease in the clutter and target responses with increasing angle of incidence). As demonstrated below for the examples considered here, $d=m / \sigma$ increases with increasing angle of incidence (although individually $m$ and $\sigma$ decrease) yielding improved detector performance. However, as $m$ and $\sigma$ decrease (near grazing) other sources of noise (e.g., receiver noise, radio-frequency interference (RFI), etc.) may become dominant, which may deteriorate detector performance in practice. Such issues are not addressed here, as we concentrate on the effects of rough-surface-induced clutter on buried-target detection. 


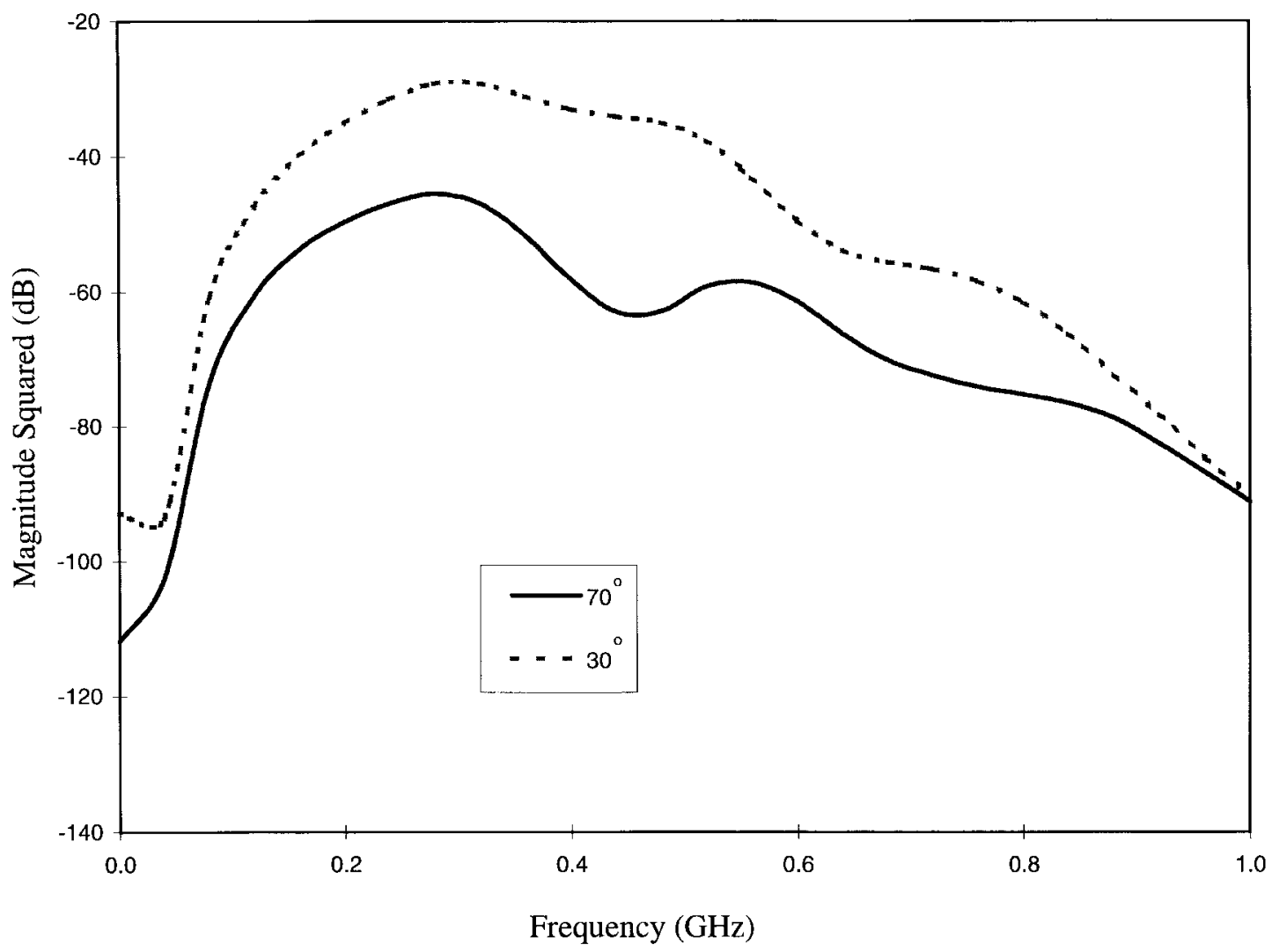

Fig. 4. Spectrum of the fields scattered from a $37.5 \mathrm{~cm} \times 12.5 \mathrm{~cm}$ lossless dielectric target $\left(\epsilon_{r}=2\right)$ buried $25 \mathrm{~cm}$ beneath a flat air-ground interface, with the soil properties $\epsilon_{r}=6$ and $\sigma=0.005 \mathrm{~S} / \mathrm{m}$. Results are plotted for angles of incidence $\theta_{i}=30^{\circ}$ and $70^{\circ}$.

\section{B. Detector Performance After Prewhitening}

We have developed a (linear-predictor) error filter (ten taps) to whiten the clutter response [37]. Using the concepts in (7) and (8), we can calculate theoretical ROC curves, under the assumption that the detector model fits the data in question. From Section III-B, the principal detector assumptions are that the target response and the clutter are additive and that the target signature is deterministic. Under these assumptions, (7) and (8) demonstrate that the whitening filter does, in theory, improve detector performance relative to the similar computations for the case of no whitening. However, in the context of our Monte Carlo simulations from which we compute simulated (empirical) ROC performance (using the FDTD computed scattered fields in which the clutter need not be additive nor the target response deterministic), we have not seen the significant whitening-induced performance enhancement predicted by (7) and (8). The degree of improvement in Monte Carlo computed ROC performance increases as the angle of incidence decreases (as the wave approaches normal incidence), but not to the degree that (7) and (8) predict (we use 300 Monte Carlo realizations for a given surface-roughness statistics).

To demonstrate whitening-induced ROC improvement as a function of incidence angle, in Fig. 5 we reconsider a $37.5 \mathrm{~cm}$ $\times 12.5 \mathrm{~cm}$ target of lossless dielectric constant $\epsilon_{r}=2$ buried $25 \mathrm{~cm}$ under the rough surface in Fig. 3. From the results in Fig. 5(a), we see that for $\theta_{i}=30^{\circ}$ the Monte Carlo simulations reveal a whitening-filter-induced ROC enhancement, which is relatively close to that found, theoretically, from (7) and (8). On the other hand, for the near-grazing case of $\theta_{i}=$ $70^{\circ}$ [Fig. 5(b)], the Monte Carlo simulations demonstrate whitening-filter-induced enhancement that is substantially less than that expected theoretically. However, it should be pointed out that the absolute Monte Carlo predicted detector quality is better near grazing $\left(\theta_{i}=70^{\circ}\right)$ than it is closer to normal incidence $\left(\theta_{i}=30^{\circ}\right)$ despite the fact that the enhancement due to prewhitening is not as great as expected theoretically.

One can understand the discrepancy between the simulated (Monte Carlo) and theoretical [(7) and (8)] ROC performance by recognizing that the field incident on the target (after transmission through the rough surface) is a random process and, therefore, so are the scattered fields. Similarly, there is additional randomization induced as the scattered fields propagate out of the soil and into air through the rough surface. One can parametrize the cumulative effect of this random transmission in and out of the soil as a random process, added onto the (deterministic) response of the target (calculated when the air-ground interface is flat). This random process need not have the same statistical properties as the fields backscattered from the rough surface (in the absence of the target) with which the whitening filter was designed (in practice, there will be surface-scatter-induced clutter and the aforementioned random-transmission-induced clutter). The mismatch between the actual clutter and that with which the whitening filter was designed will clearly deteriorate detector performance. Alternatively, instead of viewing the random transmission 


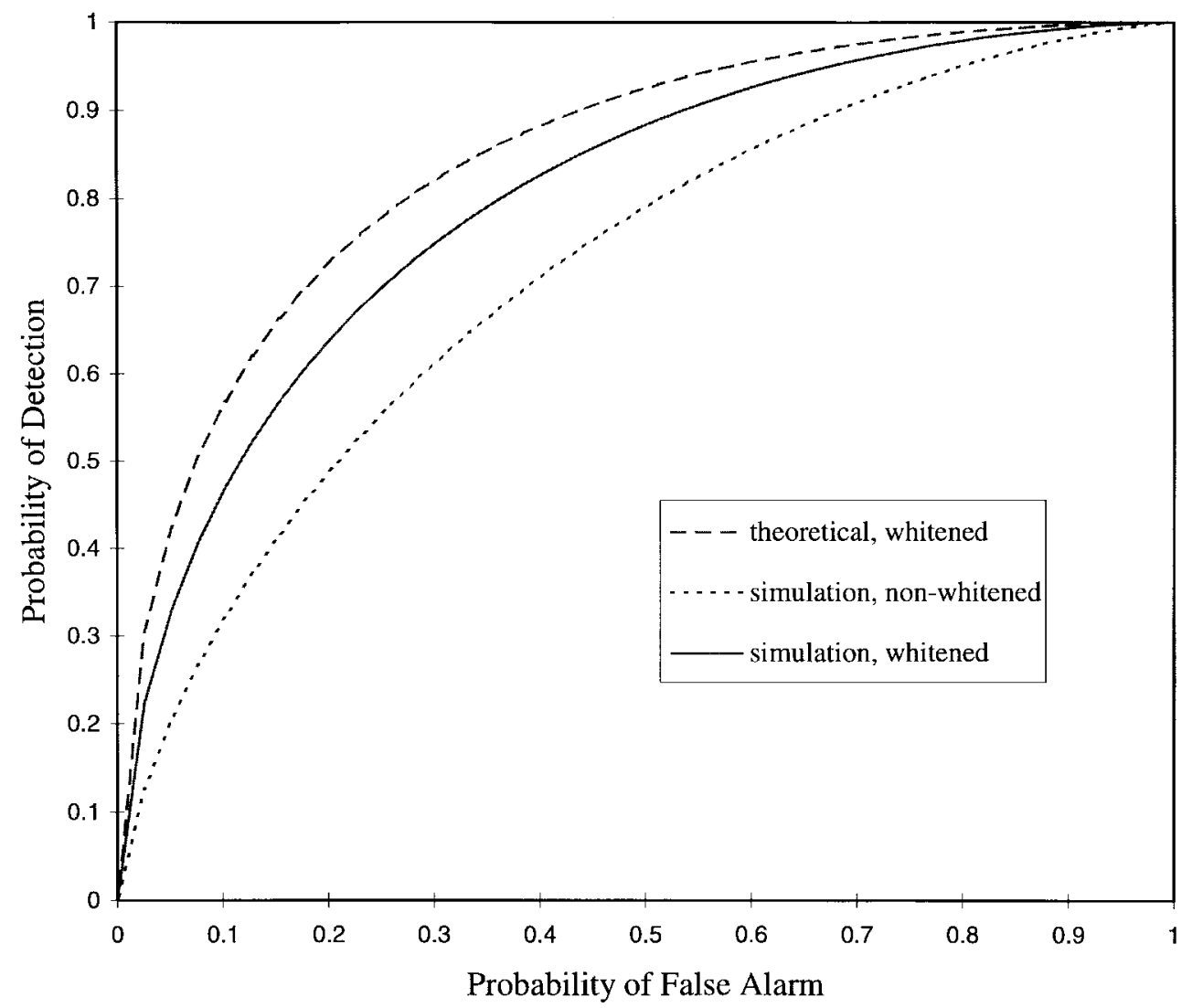

(a)

Fig. 5. Comparison of the ROC with and without prewhitening. The soil and rough air-ground interface are as in Fig. 3 and the target is a lossless dielectric $\left(\epsilon_{r}=2\right)$ of cross section $37.5 \mathrm{~cm} \times 12.5 \mathrm{~cm}$ buried $25 \mathrm{~cm}$ beneath the mean position of the interface. The theoretical ROC [20] is calculated under the assumption that the scattering data fits the detector model perfectly and the simulated ROC curves (based on 300 Monte Carlo realizations) represent actual detector performance with and without prewhitening. (a) Angle of incidence $\theta_{i}=30^{\circ}$.

through the air-ground interface as a random process added to the deterministic target signature, we can parametrize the target response itself as a random process. However, this reality runs counter to the assumptions under which our (classical) detector was designed, namely, that the target signature is deterministic (see Section III-B). In either case, the mismatch between the detector model and the physical problem is due to a randomization of the field upon entering and leaving the rough (random) air-soil interface.

For the cases considered here, the correlation length is comparable to the target width (as it often will be in practice). Therefore, the variation in the surface roughness is relatively small over the target width (the target is also relatively shallow). Thus, for near-normal incidence, the field incident upon the target and the fields backscattered from same are randomized relatively weakly, since there is modest variation (randomness) over the surface area with which these fields interact. However, as one approaches grazing the fields incident on the target interact with a wider range of the air-ground interface, sustaining more randomization (the same holds true for the backscattered fields as they are transmitted from the soil to the observer). Thus, the degree of transmission-induced randomization (upon entering and leaving the soil) increases as one approaches grazing. From the previous paragraph, it is this randomization, which is not necessarily matched to the surface clutter on which the whitening filter was designed, that causes the deterioration in detector performance. Coalescing these concepts, we can understand the reduced effectiveness of pre-whitening as the incident angle approaches grazing. However, we reiterate that in this paper, we consider one class of surface roughness, one target type, and one target depth, so the aforementioned interpretation requires further study to be stated confidently.

Finally, we reiterate that the ROC performance improves as the incidence angle approaches grazing (Fig. 5), despite the fact that the target-signature strength is smaller near grazing (Fig. 4). This implies that the variance in the clutter decreases more quickly with increasing incidence angle than does the energy in the backscattered target response. We have found this interesting result to hold for all examples considered thus far. However, in future work this phenomenon must be further tested as a function of target type and surface-roughness statistics.

In the remainder of the text, we present Monte Carlo calculated ROC curves for which the whitening filter has been utilized with the understanding that these results are inferior to those predicted by (7) and (8), with this disparity increasing as the incidence angle approaches grazing. However, these results are useful for they represent the type of performance one may expect from an actual system (the FDTD allows us to model 


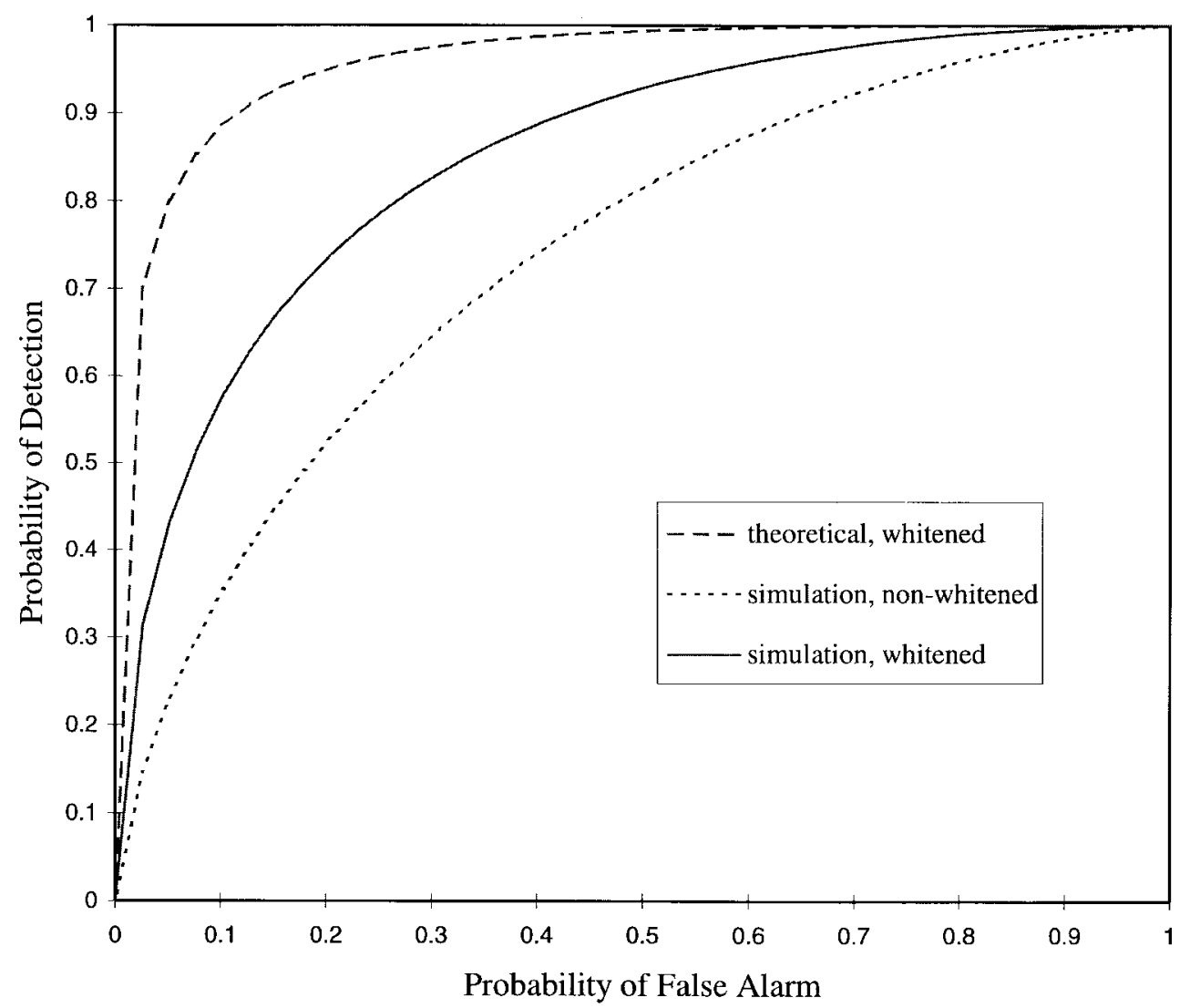

(b)

Fig. 5. (Continued.) Comparison of the ROC with and without prewhitening. The soil and rough air-ground interface are as in Fig. 3 and the target is a lossless dielectric $\left(\epsilon_{r}=2\right)$ of cross section $37.5 \mathrm{~cm} \times 12.5 \mathrm{~cm}$ buried $25 \mathrm{~cm}$ beneath the mean position of the interface. The theoretical ROC [20] is calculated under the assumption that the scattering data fits the detector model perfectly and the simulated ROC curves (based on 300 Monte Carlo realizations) represent actual detector performance with and without prewhitening. (b) angle of incidence $\theta_{i}=70^{\circ}$.

most of the relevant physics) using the type of detector often applied in practice. The type of performance demonstrated here may be inadequate for certain applications and, therefore, in Section V, we discuss the implications of the results presented here on the development of new detectors, which properly account for the physics in question.

\section{Detector Performance as a Function of Roughness Properties}

We next investigate detector performance (ROC) for a fixed target and fixed angle of incidence (near grazing), when the statistical properties of the rough surface are varied. As mentioned above, the ROC's are computed using whitening filters and in these examples the change in the surface statistics implies a different whitening filter for each example studied.

A decrease in the correlation length $l$ constitutes an increase in the variation of the rough air-ground interface and, therefore, an anticipated deterioration in detector performance. To quantify such, we consider an incident angle of $\theta_{i}=70^{\circ}$ a roughness standard deviation $h=3.95 \mathrm{~cm}$ and the same target as investigated in Figs. 4 and 5. From Fig. 6, we see that the correlation length $l=31.25 \mathrm{~cm}$ results in improved ROC performance relative to $l=18.75 \mathrm{~cm}$. Additionally, we consider an example in which the correlation length is kept fixed at $l=18.75 \mathrm{~cm}$ and rough-surface standard deviations $h=6.85 \mathrm{~cm}$ and $h=3.95 \mathrm{~cm}$ are considered. The anticipated reduction in ROC performance with increased surface variation (larger $h$ ) is demonstrated in Fig. 7.

\section{Target Properties}

In the final example, we fix the surface-roughness properties $(l=18.75 \mathrm{~cm}$ and $h=3.95 \mathrm{~cm})$ and consider an angle of incidence $\theta_{i}=70^{\circ}$. The $37.5 \mathrm{~cm} \times 12.5 \mathrm{~cm}$ lossless target is buried $25 \mathrm{~cm}$ under the mean soil height (as in all previous examples) and we consider targets with dielectric constants $\epsilon_{r}=2,3$, and 4 (recall that the soil electrical properties are characterized by $\epsilon_{r}=6$ and $\sigma=0.005 \mathrm{~S} / \mathrm{m}$ ). As with the results in Figs. 6 and 7, the qualitative ROC performance can be predicted in advance (improved detector performance with increase target-soil dielectric contrast). However, here as there, the accuracy of the FDTD computation allows us to quantify this phenomenon using the detector discussed above. In particular, the results in Fig. 8 demonstrate a marked improvement in detector performance with decreasing target dielectric constant (heightened target-soil contrast). For the surface roughness considered here, we see that detector performance is relatively poor for the case of a weak targetsoil contrast (target with $\epsilon_{r}=4$ ), underscoring the difficulty of detecting such targets with radar systems. Unfortunately, many buried mines have dielectric properties very similar to 


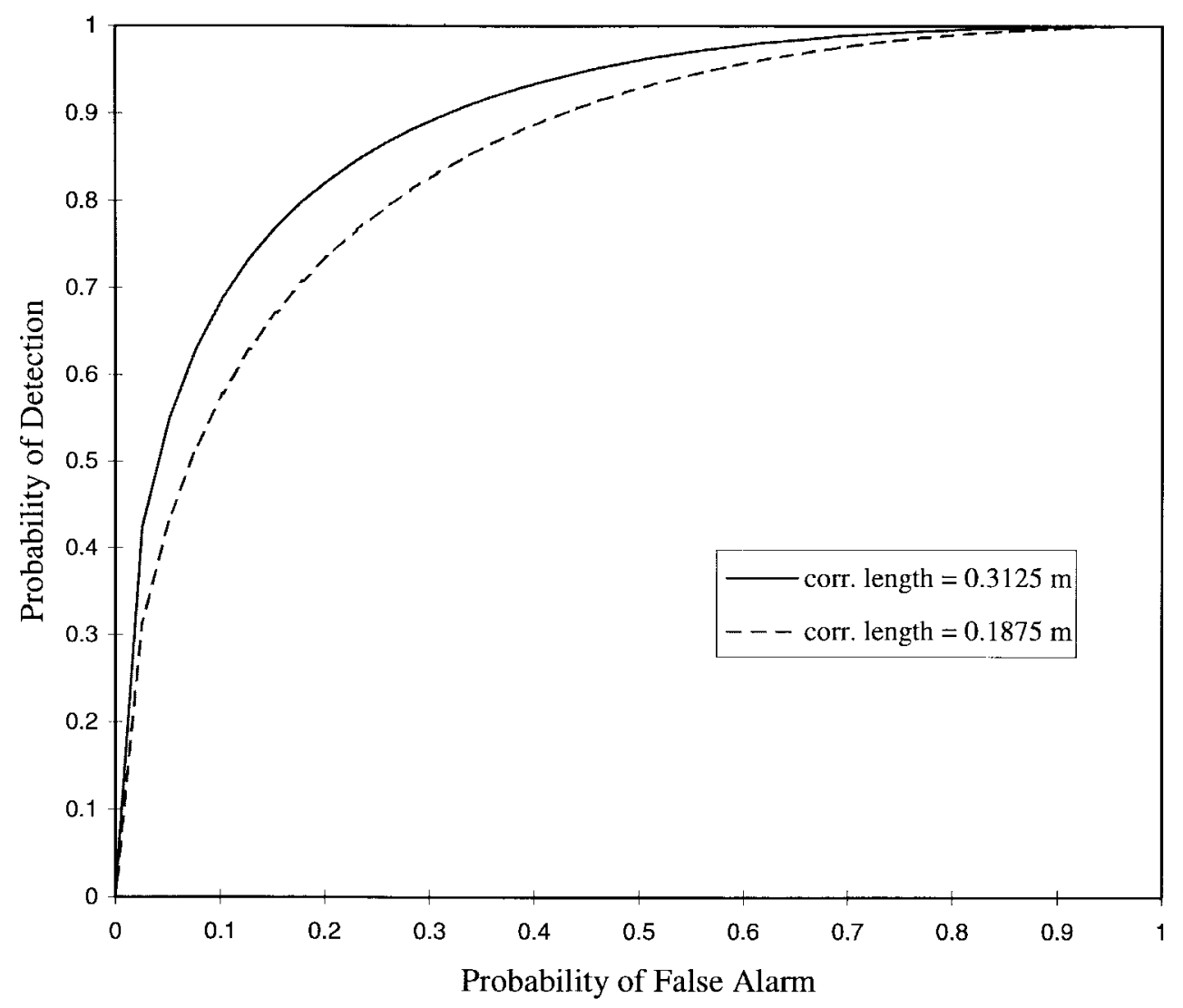

Fig. 6. Receiver operating characteristic (ROC) for a lossless dielectric target $\left(\epsilon_{r}=2\right)$ of cross section $37.5 \mathrm{~cm} \times 12.5 \mathrm{~cm}$ buried $25 \mathrm{~cm}$ beneath the mean position of the air-ground interface (soil properties: $\epsilon_{r}=6$ and $\sigma=0.005 \mathrm{~S} / \mathrm{m}$ ). The incidence angle is $\theta_{i}=70^{\circ}$ and the standard deviation of the roughness is $h=3.95 \mathrm{~cm}$. Results are plotted for the two correlation lengths $l=31.25 \mathrm{~cm}$ and $l=18.75 \mathrm{~cm}$ and the results were computed via 300 Monte Carlo iterations employing a whitening filter.

the surrounding soil [39], making the radar-based detection such a particularly challenging problem. In this case, the need for an improved detector, which properly accounts for the transmission randomness (Section IV-C), may be necessary to achieve useful system performance.

\section{CONCLUSIONS}

We have considered short-pulse electromagnetic scattering from rough (random) air-ground interfaces, with deterministic targets buried underneath (see [40] for the related problem of frequency-domain scattering; we also cite [41], which was brought to the authors attention during review). In addition to investigating the statistics of rough-surface scattering per $s e$, we have addressed processing the time-domain scattered fields for the detection of buried targets. The controlled nature of the FDTD computations used to perform this study allows a systematic investigation of the assumptions underlying conventional detectors. The matched-filter detector used here, with clutter pre-whitening, invokes the assumptions that the target response is deterministic and the clutter additive. If the theoretical and calculated (via Monte Carlo simulations) agree, then we deem these assumptions valid. When such assumptions are inappropriate, more-sophisticated detectors must be designed (or one can live with the suboptimal detector performance, if such is adequate for the application in question).
After propagating through the rough air-ground interface, the fields incident upon the target are a random process, as are the fields transmitted through the interface after scattering. Therefore, the target signature is in fact not deterministic. At issue, therefore, is the degree to which this undermines detector performance. For the examples investigated here, we have found that such a mismatch between the physics and the detector does, in fact, result in a deterioration of detector performance relative to theoretical expectations based on a perfect fit between the data and the detector. Further, the deterioration is more noticeable as one approaches grazing. An explanation of this phenomenon was given in Section IV$\mathrm{C}$, but future research is required to investigate detector performance for a wider range of surface-roughness statistics and target types.

The results of this research indicate that, under certain conditions, classical detectors may be inadequate for the important problem of detecting targets under a rough (random) interface. To ameliorate this problem, more sophisticated detectors must be developed. Detectors in which the target response is random have been developed [20]. However, such detectors incorporate conditional probability density functions, which imply a statistical analysis of the target signature. Using the FDTD, we can parametrize the statistics associated with wave propagation into and out of the soil (for the case of a random surface), the results of which can then be 


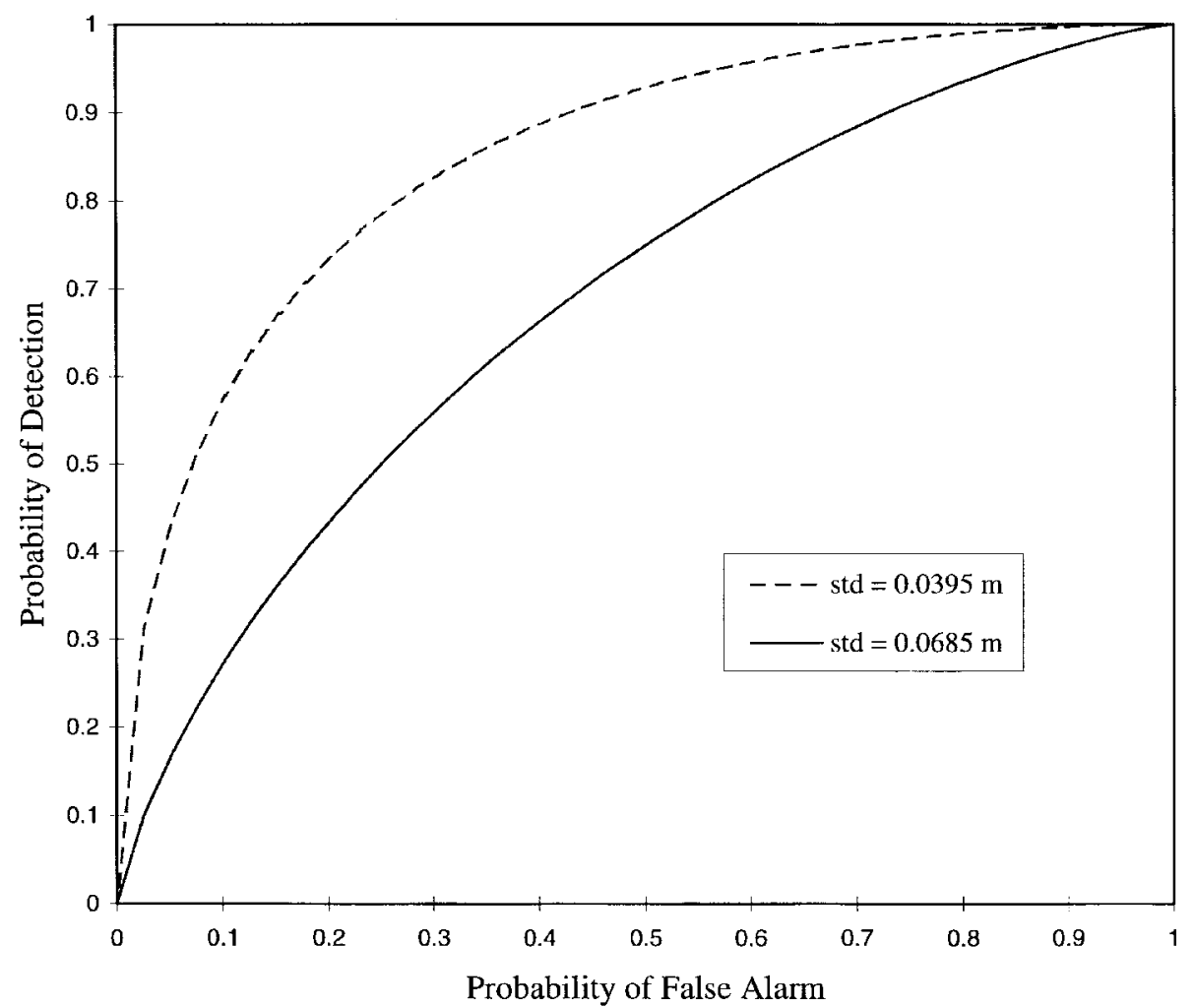

Fig. 7. Receiver operating characteristic (ROC) for the parameters in Fig. 6 except here the correlation length is fixed at $l=18.75 \mathrm{~cm}$ and the two standard deviations $h=6.85 \mathrm{~cm}$ and $h=3.95 \mathrm{~cm}$ are considered. The results were computed via 300 Monte Carlo iterations employing a whitening filter.

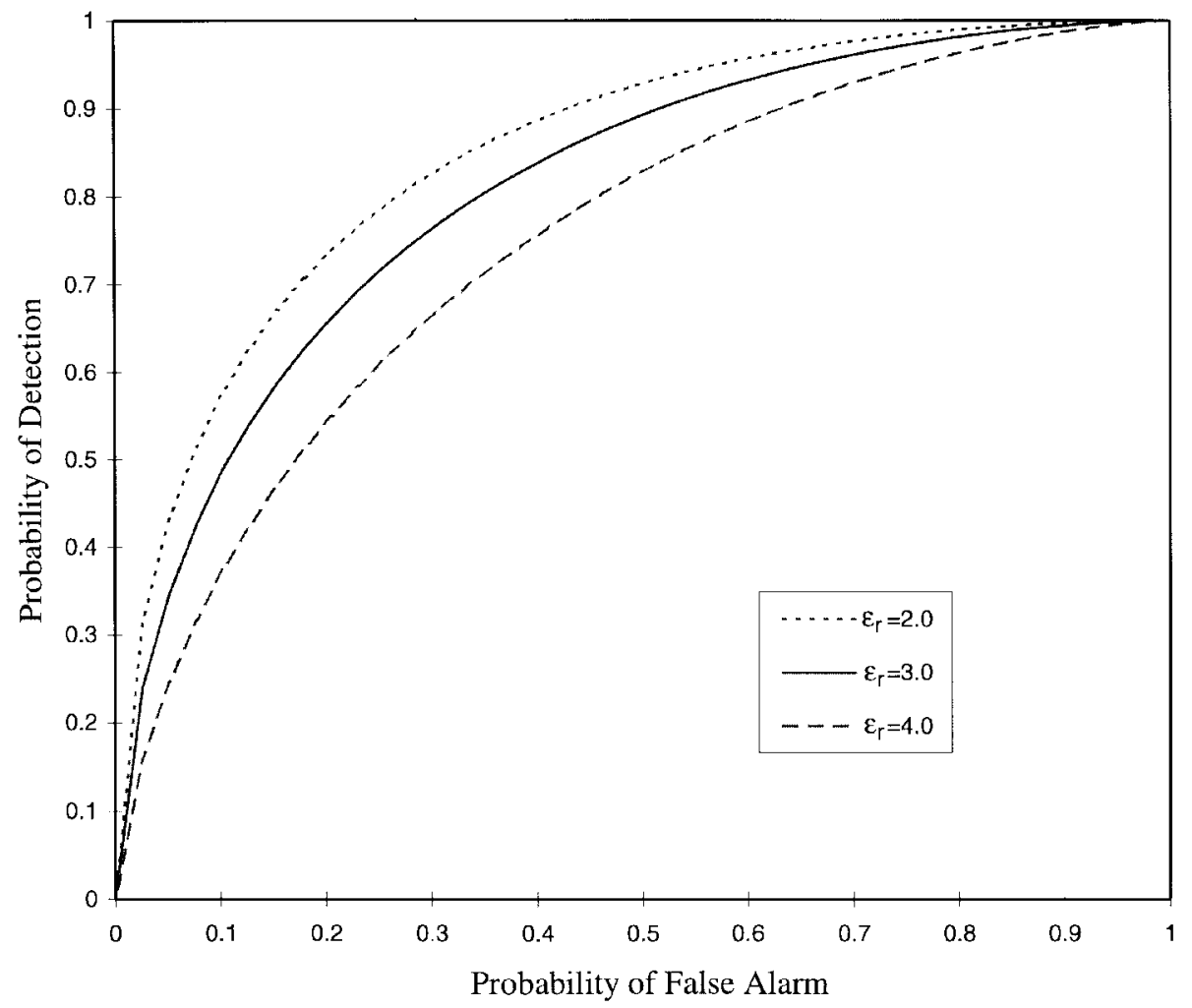

Fig. 8. Receiver operating characteristic (ROC) for a surface-roughness defined by the parameters $l=18.75 \mathrm{~cm}$ and $h=3.95 \mathrm{~cm}$ (soil properties: $\epsilon_{r}=6$ and $\sigma=0.005 \mathrm{~S} / \mathrm{m}$ ). The backscattered fields are observed for incidence angle $\theta_{i}=70^{\circ}$, the target shape and position are as in Figs. 6 and 7 , and we consider target dielectric constants $\epsilon_{r}=2,3$, and 4 . The results were computed via 300 Monte Carlo iterations employing a whitening filter. 
utilized in the context of an improved detector. This is an example wherein the statistical results from forward modeling (e.g., FDTD) can be used to improve detector performance, which is of particular importance because the measurement of such transmission statistics may be difficult (in contrast to the backscatter statistics that are measured routinely). Thus, one can measure the statistical topography of a region in question from which the transmission statistics can be modeled numerically, subsequently effecting an improved detector.

The accuracy of the FDTD allows a controlled investigation of expected detector performance for various scenarios of interest - the rough-surface scattering investigated here representing one example. Such forward modeling yields access to the fully polarimetric scattered fields, while here our detector was based on only a single polarization (a time-dependent scalar function). A fully polarimetric system, however, is far more complicated than the case considered here. Thus, it is of interest to investigate theoretically the expected improvement in ROC performance one can ideally attain if a fully polarimetric detector is effected. Thus, another important topic of future research involves polarimetric processing with the goal of quantifying ultimate detector improvements with polarimetric systems. Vector detection theory has been developed [20] and will be exploited in this context.

Finally, this paper has focused on investigating and utilizing the statistics of scattering from randomness introduced by a rough (random) air-ground interface. Inhomogeneities in the soil may also necessitate a stochastic treatment. Recall from Section I that in the case of scattering from a rough surface, the scattering itself is completely deterministic. However, the details of the rough surface change as one considers different regions of the same surface. Thus, to characterize scattering or detection in such an environment, the results must be described statistically for an ensemble of surface-roughness profiles, each characterized by the same probability distribution. By extension, rocks, roots, shrapnel, and other soil inhomogeneities also induce a deterministic scattered waveform when interrogated by electromagnetic excitation. However, the location and characteristics of such inhomogeneities can only be parametrized stochastically, resulting as well in a statistical model for the fields scattered from such environments. Thus, soil inhomogeneities give rise to another important class of clutter, which may impact the radar detection of underground targets. Moreover, as the incident wave propagates through such inhomogeneities, it will experience distortion that must be treated statistically. Therefore, the same issues addressed here with regard to surface roughness are relevant for the problem of statistical soil inhomogenities. Following the basic constructs in this paper, this will be a subject future research.

\section{ACKNOWLEDGMENT}

The authors would like to thank Duke colleagues Prof. L. Collins, Prof. J. Krolik, and Prof. L. Nolte for their helpful discussions.

\section{REFERENCES}

[1] A. Ishimaru, Wave Propagation and Scattering in Random Media. New York: Academic, 1978

[2] F. D. Hastings, J. B. Schneider, and S. L. Broschat, "A Monte-Carlo FDTD technique for rough surface scattering," IEEE Trans. Antennas Propagat., vol. 43, pp. 1183-1191, Nov. 1995.

[3] G. S. Brown, "The validity of shadowing corrections in rough surface scattering," Radio Sci., vol. 19, pp. 1461-1468, 1984.

[4] , "A new approach to the analysis of rough surface scattering," IEEE Trans. Antennas Propagat., vol. 39, pp. 943-948, July 1991.

[5] E. I. Thorso and D. R. Jackson, "The validity of the perturbation approximation for rough surface scattering using a Gaussian roughness spectrum," J. Acoust. Soc. Amer., vol. 86, pp. 261-277, 1989.

[6] L. Tsang, S. H. Lou, C. H. Chan, and A. Ishimaru, "Application of the finite element method to Monte Carlo simulations of scattering of waves by random rough surfaces with the periodic boundary condition," J. Electron. Waves Appl., vol. 5, pp. 835-855, Aug. 1991.

[7] E. I. Thorsos, "The validity of the Kirchoff approximation for rough surface scattering using a Gaussian roughness spectrum," J. Acoust. Soc. Am., vol. 83, pp. 78-92, 1988.

[8] R. Devayya and D. H. Wingham, "The numerical calculation of rough surface scattering by the conjugate gradient method," IEEE Trans. Geosci. Remote Sensing, vol. 30, pp. 645-648, Mar. 1992.

[9] R. Collin, "Electromagnetic scattering from perfectly conducting rough surfaces using a new full-wave method," IEEE Trans. Antennas Propagat., vol. 40, pp. 1466-1477, Dec. 1992.

[10] S. Vitebskiy and L. Carin, "Moment-method modeling of short-pulse scattering from and the resonances of a wire buried inside a lossy, dispersive half space," IEEE Trans. Antennas Propag., vol. 43, pp. 1303-1312, Nov. 1995.

[11] S. Vitebskiy, K. Sturgess, and L. Carin, "Short-pulse scattering from buried perfectly conducting bodies of revolution," IEEE Trans. Antennas Propagat., vol. 44, pp. 143-151, Feb. 1996.

[12] S. Vitebskiy and L. Carin, "Resonances of perfectly conducting wires and bodies of revolution buried in a lossy, dispersive half space," IEEE Trans. Antennas Propagat., vol. 44, pp. 1575-1583, Dec. 1996.

[13] L. Peters, J. J. Daniels, and J. D. Young, "Ground penetrating radar as a subsurface environmental sensing tool," Proc. IEEE, vol. 82, pp. 1802-1822, Dec. 1994.

[14] J. M. Bourgeois and G. S. Smith, "A fully three-dimensional simulation of a ground-penetrating radar: FDTD theory compared with experiment," IEEE Trans. Geosci. Remote Sensing, vol. 34, pp. 36-44, Jan. 1996.

[15] P. E. Wannamaker, G. W. Hohmann, and W. A. San Filipo, "Electromagnetic modeing of three-dimensional bodies in layered earths using integral equations," Geophys., vol. 49, pp. 60-74, Jan. 1984.

[16] D. L. Moffatt and R. J. Puskar, "A subsurface electromagnetic pulse radar," Geophys., vol. 41, pp. 506-518, June 1976.

[17] S. Vitebskiy, L. Carin, M. Ressler, and F. Le, "Ultra-wideband, shortpulse ground-penetrating radar: Simulation and measurement," IEEE Trans. Geosci. Remote Sensing, to be published.

[18] M. A. Ressler and J. W. McCorkle, "Evolution of the Army Research Laboratory ultra-wideband test bed," in Ultra-Wideband Short-Pulse Electromagnetics-2, L. Carin and L. B. Felsen, Eds. New York: Plenum, 1995, pp. 109-123.

[19] S. L. Earp, E. S. Hughes, T. J. Elkins, and R. Vickers, "Ultra-wideband ground-penetrating radar for the detection of buried metallic mines," IEEE Aerosp. Electron. Syst. Soc. Mag., pp. 30-34, 1996.

[20] H. L. Van Trees, Detection, Estimation, and Modulation Theory. New York: Wiley, 1968.

[21] K. S. Yee, "Numerical solution of initial boundary value problems involving Maxwell s equations in isotropic media," IEEE Trans. Antennas Propagat., vol. AP-14, pp. 302-307, May 1966.

[22] K. S. Kunz and R. J. Luebbers, The Finite-Difference Time-Domain Method for Electromagnetics. Boca Raton, FL: CRC, 1993.

[23] A. Taflove, Computational Electrodynamics: The Finite-Difference Time-Domain Method. Norwood, MA: Artech House, 1995.

[24] D. E. Merewether, R. Fisher, and F. W. Smith, "On implementing a numerical Huygens surface in a finite difference program to illuminate scattering bodies," IEEE Trans. Nucl. Sci., vol. NS-27, pp. 1829-1833, Dec. 1980

[25] T.-T. Hsu and L. Carin, "FDTD analysis of plane-wave diffraction from microwave devices on an infinite dielectric slab," IEEE Microwave Guided Wave Lett., vol. 6, pp. 16-18, Jan. 1996.

[26] K. Demarest, Z. Huang, and R. Plumb, "An FDTD near-to-far-zone transformation for scatterers buried in stratified grounds," IEEE Trans. Antennas Propagat., vol. AP-44, pp. 1150-1157, Aug. 1996. 
[27] J. P. Berenger, "A perfectly matched layer for the absorption of electromagnetic waves," J. Comput. Phys., vol. 114, pp. 185-200, Oct 1994.

[28] J. Fang and Z. Wu, "Generalized perfectly matched layer an extension of Berengers perfectly matched layer boundary condition," IEEE Microwave Guided Wave Lett., vol. 5, pp. 451-453, Dec. 1995.

[29] Z. Wu and J. Fang, "Numerical implementation and performance of perfectly matched layer boundary condition for waveguide structures,' IEEE Trans. Microwave Theory Tech., vol. 43, pp. 2676-2683, Dec. 1995.

[30] R. L. Higdon, "Absorbing boundary conditions for difference approximations to the multi-dimensional wave equations," Math. Comput., vol. 47, no. 176, pp. 437-459, Oct. 1986.

[31] L. B. Felsen and N. Marcuvitz, Radiation and Scattering of Waves. Englewood Cliffs, NJ: Prentice-Hall, 1973.

[32] L. B. Felsen and F. Niu, "Spectral analysis and synthesis options for short-pulse radiation from a point dipole in a grounded dielectric layer," IEEE Trans. Antennas Propagat., vol. 41, pp. 747-754, June 1993.

[33] P. G. Petropoulos, "Stability and phase error analysis of FDTD in dispersive dielectrics," IEEE Trans. Antennas Propagat., vol. 42, pp. 62-69, Jan. 1994.

[34] P. G. Petropoulos, "Phase error control for FDTD methods of second and fourth order accuracy," IEEE Trans. Antennas Propagat., vol. 42 , pp. 859-862, June 1994.

[35] A. Papoulis, Probability, Random Variables, and Stochastic Processes, 2nd ed. New York: McGraw-Hill, 1984.

[36] L. Franks, Signal Theory. Englewood Cliffs, NJ: Prentice-Hall, 1969.

[37] S. Haykin, Adaptive Filter Theory, 3rd ed. Englewood Cliffs, NJ: Prentice-Hall, 1996.

[38] J. E. Hipp, "Soil electromagnetic parameters as functions of frequency, soil density, and soil moisture," Proc. IEEE, vol. 62, pp. 98-103, Jan. 1974.

[39] A. Dubey, I. Cindrich, J. M. Ralston, and K. Rigano, Eds., "Detection technologies for mines and minelike targets," in SPIE Proc., Orlando, FL, Apr. 1995, vol. 2496.
[40] K. O’Neil, R. F. Lussky, Jr., and K. D. Paulsen, "Scattering from a metallic object embedded near the randomly rough surface of a lossy dielectric," IEEE Trans. Geosci. Remote Sensing, vol. 34, pp. 367-376, Mar. 1996.

[41] Y. Miyazaki, "Statistical refelection properties of an electromagnetic pulse by buried objects in random media using the FDTD," in Proc. Int. Symp. Antennas Propagat., Chiba, Japan, Sept. 1996.

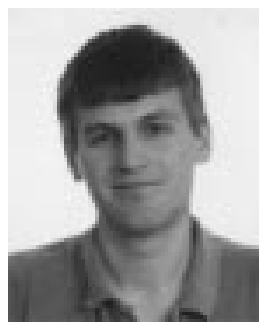

Traian Dogaru (S'96) was born in Bucharest, Romania, in 1966. He received the Engineering degree from the Polytechnic University of Bucharest, Romania, in 1990, and the M.S. degree in electrical engineering from Duke University, Durham, NC. $\mathrm{He}$ is currently working toward the Ph.D. degree at the same university.

Between 1992-1995, he held different engineering positions in the magnetic recording industry. His main research interests are in electromagnetic wave theory, computational electromagnetics, and radar-related signal processing.

Lawrence Carin (SM'96) was born on March 25, 1963, in Washington, DC. He received the B.S., M.S., and Ph.D. degrees in electrical engineering at the University of Maryland, College Park, in 1985, 1986, and 1989, respectively. In 1989, he joined the Electrical Engineering Department at the Polytechnic University, Brooklyn, NY, as an Assistant Professor and became an Associate Professor there in 1994. In September 1995 he joined the Electrical Engineering Department at Duke University, Durham, NC, where he is an Associate Professor. His current research interests include quasi-planar transmission lines, short-pulse scattering and propagation, and signal processing.

Dr. Carin is a member of the Tau Beta Pi and Eta Kappa Nu honor societies. 